\title{
PRIORITIZED BUFFER MANAGEMENT IN PHOTONIC PACKET SWITCHES FOR DIFFSERV ASSURED FORWARDING
}

\author{
Hiroaki Harai ${ }^{1}$ and Masayuki Murata ${ }^{2}$ \\ ${ }^{1}$ Communications Research Laboratory, Konanei-shi, Tokyo 184-8795, Japan \\ harai@crl.go.jp \\ ${ }^{2}$ Osaka University, Toyonaka-shi, Osaka 560-0043, Japan \\ murata@cmc.osaka-u.ac.jp
}

Abstract We study photonic packet switching architecture that enables a high node throughput and provides priority services in DiffServ (Differentiated Services). As we will show in this paper, a photonic packet switch can be equipped with an ultrahigh-speed address-lookup capability in the optical domain. However, the optical buffer must be managed in the electrical domain. In this paper, we investigate buffer management schemes with less complexity, such that the capability of the ultra-high-speed packet forwarding can be sustained in the photonic switch. We propose an implementation method for PBS (Partial Buffer Sharing) that provides DiffServ AF (Assured Forwarding). We show that PBS is applicable to single-stage optical buffer. Also, we propose a new buffer management method, called PBSO (PBS with Overwriting), which is applicable to broadcast-andselect-based single-stage optical buffer. PBSO is also applicable to the current space-switch-based single-stage optical buffer with an addition of a few optical components. The PBS and PBSO methods are based on a single queue and their complexities are $O(1)$ and $O(p)$, respectively, where $p$ is the number of levels of drop precedence. Through simulation study, we show that both methods provide different levels of drop precedence for DiffServ AF, and that PBSO improves buffer utilization and the packet loss probability of both drop-level packets.

Keywords: Photonic packet switch, buffer management, partial buffer sharing with overwriting, single queue, DiffServ, level of drop precedence

\section{Introduction}

Because of the spread of multimedia systems such as WWW, the Internet should handle a greater amount of traffic than before. With the spread of highspeed access services such as ADSL (Asymmetric Digital Subscriber Line), the increase in the Internet traffic is now accelerating even more. The acceleration in access networks affects the design of the core network. In order to build 
broadband core networks capable of handling a tremendous amount of traffic, it is necessary to improve a packet forwarding capability (i.e., node throughput) at a node as well as to increase the link capacity. Currently, we rely on electronic processing for packet forwarding. In this case, the node throughput can be improved by an advancement in the LSI technology known as Moore's Law and large-scale distributed/pipelined processing. While the link capacity is easily increased by bundling optical fibers, the integration and pipelined processing may limit the increase in the node throughput. Thus, as the line speed and the number of ports at the node are increasing, electrical limitations motivate us to introduce optical technology into packet forwarding.

Several researchers investigated photonic packet switches/routers (e.g., $[8,6$, $2,15,11])$, and some have introduced an optical buffer $[8,6,2]$. The functions of packet switches are roughly divided into the following five functions: (1) address/label lookup (i.e., forwarding), (2) switching, (3) buffer management, (4) buffering, and (5) routing functions. A packet switch having functions (2) and (4) in the optical domain is usually called a photonic packet switch, and it requires optical switches and optical buffers. The photonic packet switch forwards packets without $\mathrm{O} / \mathrm{E}$ or $\mathrm{E} / \mathrm{O}$ conversion with functions (2) and (4); it can make use of the broadband property of an optical signal. Moreover, improving function (1) is promising because high-speed processing can be achieved by optical analog processing such as multi-wavelength label processing [15] and optical code label processing [11]. By combining these types of processing with optical switches, we can obtain a very high-performance $1 \times N$ photonic packet switch. A packet switch having functions (1), (2), and (4) in the optical domain is called a photonic packet switch in this paper.

To achieve a higher throughput in the photonic packet switch, (3) buffer management must also be improved. Since optical RAM (Random Access Memory) is still impractical, the function (3) still requires electronic processing in order to determine the delay in the optical buffer, which is necessary to avoid packet collision. It is therefore important to use a less complex buffer management algorithm for the photonic packet switch because a complex algorithm cannot handle a large number of packets that arrive simultaneously. That is, the use of a complicated algorithm may result in an unexpected degradation of the photonic packet switch performance.

In the Internet, we easily notice the diversification of applications as well as an increase in the amount of traffic. The diversification of applications implies the need for priority services in order to provide high-quality services rather than best-effort services. DiffServ (Differentiated Services) [1] is a typical example. Priority queueing is a practical method for DiffServ. Since advances in the LSI technology have alleviated constraints on the buffer size in the electrical domain, multiple queues such as those for CBQ (Class-Based Queueing) [5] and DRR (Deficit Round Robin) [14] are current fashion for DiffServ. We 
cannot expect such elegant and complicated queue management schemes in the photonic packet switch, but we can realize a capability of DiffServ with sustaining high node throughput, which is the main subject of this paper.

In this paper, we investigate buffer management methods in a photonic packet switch. We pose two constraints: achieving less complexity for high-throughput and providing different levels of drop precedence in DiffServ AF (Assured Forwarding) [7]. For this purpose, we first consider the differences between an optical buffer and an electronic buffer. Optical buffer is composed of multiple FDLs (optical Fiber Delay Lines) and optical switches while an electronic buffer is composed of RAM. The size of optical buffer is limited because switching and filtering degrade optical signals. Moreover, multiple queues are not suitable for the FDL buffer due to an essential nature of FDL, as will be described later. We therefore focus on the priority-based buffer management using a single queue. We introduce PBS (Partial Buffer Sharing) [13] method for the buffer management suitable for the optical buffer. The complexity of this method is small, that is, the algorithm complexity is $O(1)$, leading a packet switch to high throughput. We propose a realization method for the priority-based buffer management of PBS in two optical buffer architectures. To improve buffer utilization, we newly propose PBSO (Partial Buffer Sharing with Overwriting) by extending PBS. The complexity of PBSO is $O(p)$, where $p$ is the number of levels of drop precedence. Since $p$ is much smaller than the maximum queue length in our case, the complexity is negligible. We also propose a realization method for the priority-based buffer management of PBSO in two optical buffer architectures. The performance of the PBS and PBSO strategies will be examined through simulation experiments.

This paper is organized as follows. In Section 2, we briefly summarize the photonic packet switch architecture. In Section 3, we describe a method for the management of an optical buffer that provides different levels of drop precedence for DiffServ AF. PBS is introduced for this purpose. We also propose the PBSO strategy. In Section 4, we investigate optical buffer architecture for buffer management of PBS and PBSO. Section 5 is devoted to performance evaluation of PBS and PBSO strategies. We present our conclusions in Section 6.

\section{Photonic Packet Switch Architecture}

Figure 1 depicts photonic packet switch architecture to which our buffer management of optical buffer is applied. The $N \times N$ packet switch consists of the number $N$ of $1 \times N$ bufferless packet switches followed by the number $N$ of $N \times 1$ buffers. Every pair of a $1 \times N$ switch and an $N \times 1$ buffer is optically interconnected in a full meshed manner. The $1 \times N$ bufferless packet switches make the address lookup function fast by applying photonic address lookup functions $[15,11]$ to the packet switch. As a result, the architecture provides 


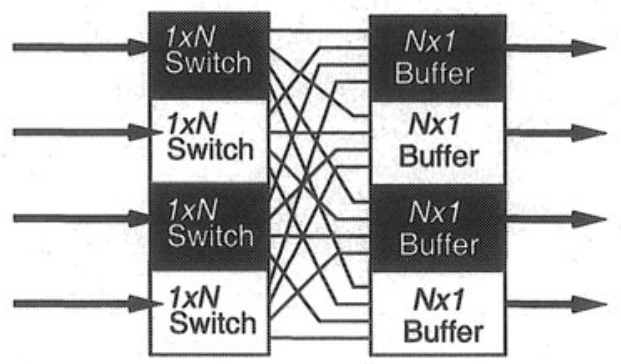

Figure 1. An $N \times N$ photonic packet switch architecture $(N=4)$

an ultra-high node throughput to the packet switch. The $N \times 1$ buffers are next attached. This is used to avoid the collision of packets and to improve packet loss probability. Each buffer is allocated to a different output of the $N \times N$ packet switch.

The existing optical buffer consists of multiple FDLs, each of which has a different length $[8,6,2,9,4,10]$. These buffers can avoid internal collision of packets with the help of buffer management that assigns each packet to a different FDL. This is similar to packet buffering in an electronic RAM buffer, which can avoid internal contention by allocating multiple data to different entries. However, these two buffers are significantly different. The electronic RAM buffer can dequeue packets at any time, while in the optical FDL buffer, the departure time of a packet is determined by the length of each FDL in the buffer. To avoid packet collision, we need a buffer management method in which the appropriate FDL is selected for each arriving packet before the packet enters the FDL buffer. Also, to obtain a priority service for DiffServ $\mathrm{AF}$, we need additional buffer management, which we will describe in the next section.

\section{Optical Buffer Management using a Single Queue}

In this section, we first describe prioritized buffer managements in an electronic buffer, and point out the inappropriateness of its direct extension to an optical buffer. We then propose prioritized buffer management for optical buffer based on a new mechanism.

Some IP routers and/or ATM switches support the buffer management of multiple queues, where one buffer is divided into multiple buffers and each is dedicated to a single queue. FQ (Fair Queueing) [3], CBQ (Class-Based Queueing) [5], and Per-Flow Queueing are examples. Multiple-queue methods for electronic buffers are effective for priority queueing because each queue is managed individually, and one scheduler dequeues one packet from the corresponding queue at a time. However, the buffer utilization is degraded when compared to that of a single queue because the buffer is completely partitioned. 


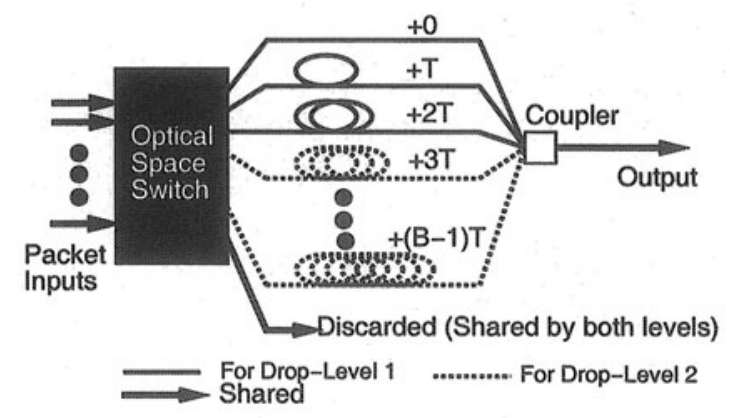

Figure 2. An example of multiple queues

This problem is alleviated with the advances in the LSI technology that make the electronic buffer bigger and bigger.

On the other hand, it is inappropriate to maintain multiple queues in the optical buffer due to the nature of FDL. Now, we consider two methods. In the first method, each drop-level packet is allowed to enter a fraction of FDLs and FDLs are completely partitioned according to the number of drop levels. Figure 2 depicts an example; drop-level 1 packets (with higher priority) are allowed to enter shorter FDLs while drop-level 2 packets (with lower priority) enter longer FDLs. Two queues are virtually composed for each drop level. Drop-level 2 packets enter longer FDLs only if their queue (i.e., longer part of FDLs) is not full. All drop-level 2 packets are given a longer delay even if another queue with shorter FDLs is empty. In contrast, drop-level 1 packets may not be allowed to enter their queue even if their queue is not full. Droplevel 1 packets are affected by another queue. For example, when a drop-level 2 packet reaches the head of the queue and is transmitted at the output port while a drop-level 1 packet arrives, assigning the drop-level 1 packet to the first FDL $\left({ }^{66}+0\right.$ " in Fig. 2) generates collision at the output port. To avoid the collision, the drop-level 1 packet is allowed to enter the second FDL (" $+T$ " in Fig. 2) even if the queue is empty. This indicates that even if FDLs are partitioned, the chance of a prior packet entering empty position in the queue depends on the position of packets with another drop level. Thus, the above method is inappropriate for handling multiple queues.

In the other method, two optical buffers are completely partitioned, and each is dedicated to one level of drop precedence. Although this method can reduce the delay for drop-level 2 packets, the above dependency still remains. It is thus inappropriate for handling multiple queues.

In this section, we investigate priority-based buffer managements of optical buffers using a single queue. The following two strategies provide different levels of drop precedence for DiffServ AF. 


\subsection{PBS: Partial Buffer Sharing}

The first strategy is PBS (Partial Buffer Sharing) [13]. PBS is a simple $O(1)$ strategy based on a single queue, which enables high-speed buffer management of the optical buffer. In the PBS strategy, the threshold value should be predetermined. When the queue is shorter than the threshold, every packet is allowed to enter the queue. When the queue length is equal to or longer than the threshold, only prior packets (which we will refer to as drop-level 1 packets) are allowed to enter the queue while low-priority (drop-level 2) packets are discarded. Since no loop operation is needed, $O(1)$ is achieved.

The buffer utilization in the PBS strategy is lower than that in other singlequeue-based strategies such as HOL (Head-of-the-Line priority queueing) [12] and PO (Push Out) [13]. Since HOL and PO allow using the whole buffer, the utilization of the optical buffer increases. In the HOL strategy, when a drop-level 1 packet arrives, the packet enters the queue ahead of all drop-level 2 packets. In the PO strategy, when the buffer is full and a drop-level 1 packet arrives, the packet pushes out the drop-level 2 packet that entered the queue most recently. All drop-level 1 packets behind the discarded drop-level 2 packet are moved ahead. These mean that HOL and PO have the $O(B)$ complexity where $B$ is the maximum queue length (i.e., the number of FDLs). This complexity apparently limits the speed of buffer management. In order to develop a photonic packet switch with a high line speed, simpler PBS is more effective than the more complicated HOL and PO.

\subsection{PBSO: Partial Buffer Sharing with Overwriting}

PBS provides different levels of drop precedence of packets in the optical buffer at the expense of buffer utilization. Of course, improving the buffer utilization is important. We thus propose PBSO (PBS with Overwriting), which can improve the buffer utilization. Its complexity is $O(p)$, where $p$ is the number of levels of drop precedence. Since $p$ is generally small, the complexity is negligible.

Under the PBSO (PBS with Overwriting) strategy, the buffer manager allows every arriving packet to enter the queue when the queue is shorter than the threshold as in the PBS strategy (see also Fig. 3). When the queue length is equal to or greater than the threshold, an arriving drop-level 2 packet is allowed to enter the tail of the queue. The behavior to drop-level 1 packets is the same as PBS. Namely, an arriving drop-level 1 packet is allowed to enter the queue at the next position of the drop-level 1 packet that entered the queue most recently if the drop-level 1 packet is located behind the threshold. Otherwise, the droplevel 1 packet enters the queue at the position of the threshold. In this case, the drop-level 1 packet may overwrite a drop-level 2 packet if the drop-level 2 packet is located after the threshold. The overwritten drop-level 2 packet is 
(a)

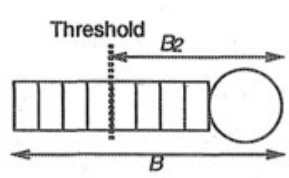

(c)

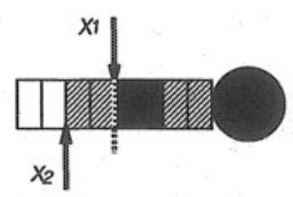

(b)

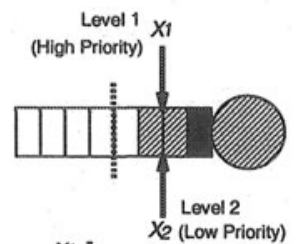

(d)

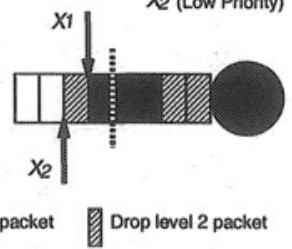

Figure 3. A behavior of PBSO

regarded as a discarded packet. The behavior of PBSO can be represented by two variables, $X_{1}$ and $X_{2}$.

Hereafter, we assume that a packet is of a fixed length and each packet arrives at a photonic packet switch synchronously. At most $N$ packets arrive at the optical buffer and the buffer manager must handle all the packets within the time equivalent to the packet length, $T$.

Now, we will describe the behavior to packets in the PBSO strategy.

- The buffer manager handles packets from multiple ports by following round robin scheduling. It takes time $T$ to handle all the ports.

-1 The buffer manager introduces $X_{1}$ and $X_{2}$ to represent where drop-level 1 and drop-level 2 packets are stored in the queue, respectively.

- When a packet arrives at a port, buffer manager stores the packet according to $X_{1}$ and $X_{2}$. However, an arriving drop-level 1 packet is discarded if $X_{1}=B$, and an arriving drop-level 2 packet is discarded if $X_{2}=B$. After the packet has been handled, $X_{1}$ and $X_{2}$ change as follows.

- If the queue is shorter than threshold $B_{2}$, set $X_{1} \leftarrow X_{1}+1$ and $X_{2} \leftarrow X_{2}+1$ regardless of the drop level of the arriving packet.

- If the queue length is equal to or longer than the threshold and if a drop-level 2 packet arrives, set $X_{2} \leftarrow X_{2}+1$.

- If the queue length is equal to or longer than the threshold and if a drop-level 1 packet arrives, set $X_{1} \leftarrow X_{1}+1$. A position for drop-level 2 packet is then handled not to be ahead of a position for drop-level 1 packet, that is, set $X_{2} \leftarrow \max \left(X_{1}, X_{2}\right)$.

- If $X_{1}=B$ or $X_{2}=B$, the corresponding variables do not change. 
After the packets of all ports have been handled, the queue length decreases by one. This is because one packet departs from the queue. At this time,

- If the queue is equal to or shorter than the threshold, set $X_{1} \leftarrow$ $X_{1}-1$ and $X_{2} \leftarrow X_{2}-1$.

- Otherwise, set $X_{1} \leftarrow \max \left(X_{1}-1, B_{2}\right)$ and $X_{2} \leftarrow X_{2}-1$.

Figure 3 illustrates a behavior of PBSO. Figure 3(a) represents the relationship between the maximum queue length $(B)$ and the threshold value $\left(B_{2}\right)$. Let us consider that a queue whose length is four, that is, one packet is served and three packets wait as in Fig. 3(b). Now, five packets (drop-levels 1, 1, 2, 2, 1 in the scheduled order) arrive at the packet switch, where all the packets are designated to the same output port. Variables $X_{1}$ and $X_{2}$ for the new packets are as in the figure since the tail of the packets shown in Fig. 3(b) move ahead after time $T$.

Now, we will descrive how the five arriving packets are handled. The first two drop-level 1 packets are stored as in Fig. 3(c) since the queue is shorter than the threshold at this time. When the next two drop-level 2 packets are handled, the queue length reaches the threshold. The drop-level 2 packets are allowed to enter the tail of the queue in order. Figure 3(c) shows $X_{1}$ and $X_{2}$ in the transient state. The remaining drop-level 1 packet enters the queue at the position as in Fig. 3(d). Consequently, one drop-level 2 packet is overwritten by one drop-level 1 packet.

\section{Optical Buffer Architecture for Buffer Management of PBS and PBSO}

We investigate optical buffer architectures for PBS and PBSO providing different levels of drop precedence in DiffServ AF. Several optical buffer architectures have been proposed. These are categorized into three: a single-stage buffer architecture, in which a number of FDLs are allocated in parallel and a packet goes through one of them [8,2,9], a multi-stage buffer architecture, in which a number of single-stage buffers are connected in tandem $[8,6,9,10]$, and a recursive buffer architecture, in which a FDL may be used for a packet several times by loopback interconnection $[8,9,4]$. Generally speaking, a multistage buffer and a recursive buffer have internal blocking without the used of a complex architecture and management (e.g., [10]) while they have a smaller number of FDLs compared to a single-stage buffer. In contrast, a single-stage buffer does not have internal blocking, which results in easy implementation of PBS and PBSO. We focus on the buffer management of PBS and PBSO in a single-stage buffer. 


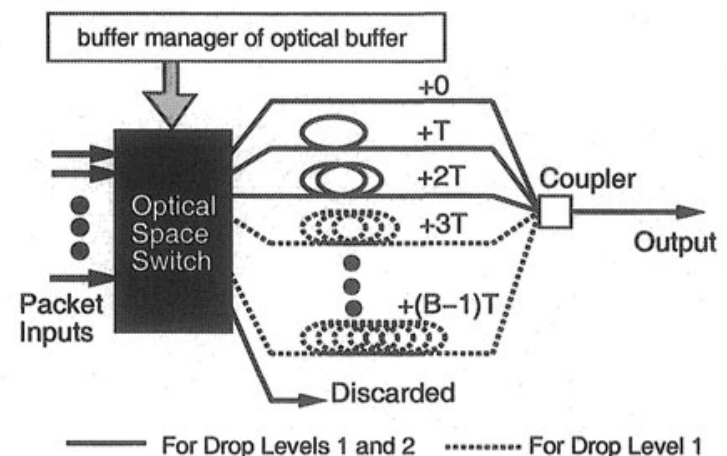

Figure 4. A space-switch-based single-stage optical buffer for PBS strategy

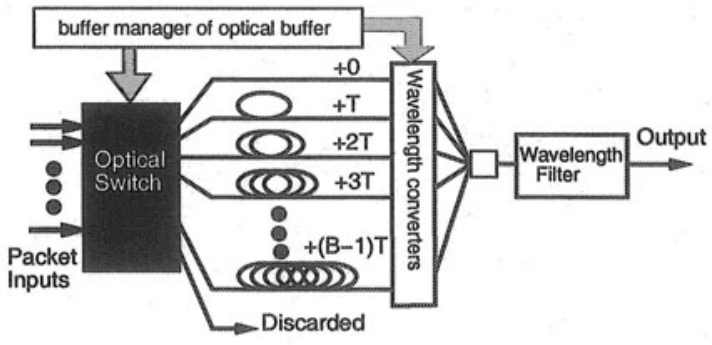

Figure 5. A space-switch-based single-stage optical buffer for PBSO strategy

There are two types of single-stage buffers: a space-switch-based buffer and a broadcast-and-select-based one. Figure 4 shows a space-switch-based singlestage buffer. The output ports of the space switch are connected to different FDLs. Buffer management is performed by controlling the optical switch. For this purpose, every signal that indicates a packet arrival must reach the buffer manager $T$ time before the corresponding packet arrives at the optical buffer. The buffer manager handles at most $N$ packets within time $T$. Wavelength conversion may be used to increase the size of the buffer $[6,2]$ and replace the optical space switch with AWG (Arrayed Waveguide Grating) [8].

The buffer management of the PBS strategy is applicable to the above spaceswitch-based single-stage buffer. A buffer manager handling PBS strategy is required. The space switch can discard drop-level 2 packets arriving when the queue length is equal to or longer than the threshold. When the threshold is set at $B_{2}=3$, the FDLs shown by the dotted lines in Fig. 4 are dedicated to drop-level 1 packets.

PBSO cannot be used for the existing space-switch-based buffer. This is because packets cannot be extracted from the buffer. This prevents the buffer manager from overwriting drop-level 2 packets. We therefore propose two optical buffer architectures by extending the space-switch-based buffer. In the first one, a space switch is added. The space switch is inserted between FDLs 


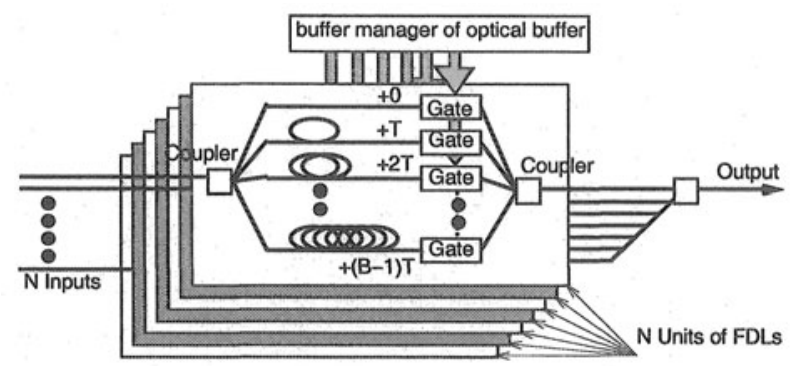

Figure 6. A broadcast-and-select based single-stage optical buffer for PBS and PBSO strategies

and the coupler in Fig. 4. The PBSO strategy is achieved by making the added optical switch discard overwritten drop-level 2 packets. In the other one, we introduce wavelength converters. Figure 5 shows the optical buffer architecture for the PBSO strategy. The wavelength of overwritten drop-level 2 packets is changed to a wavelength not used in the link. The following wavelength filter does not pass the packets.

Figure 6 depicts a broadcast-and-select single-stage buffer, in which arriving packets are split and the split packets enter all FDLs. Buffer management is performed by controlling the optical gates. If the buffer manager gives a $k T$ delay to an arriving packet, it only opens the $(k+1)$ th gate in $k T$ time $\left({ }^{66}+k T\right.$ " in Fig. 6). Only packets designated to the output port are passed through the corresponding gate. Since a unit of FDLs has one input, $N$ units equivalent to the number of input ports of the packet switch are required to compose an $N \times 1$ buffer.

The buffer management of the PBS strategy is applicable to a broadcast-andselect buffer because the buffer manager does not open any gate for drop-level 2 packets that arrived when the queue length was equal to or longer than the threshold. More notably, PBSO is applicable to the optical buffers without any extension. This is because overwritten drop-level 2 packets are still in the FDLs when the buffer manager decides on their discarding.

\section{Performance of PBS and PBSO Strategy}

In this section, we investigate the performance of the PBS and PBSO strategies. We first show that both strategies can provide different levels of drop precedence in DiffServ AF. We then show that PBSO improves buffer utilization more than does PBS. It also improves the packet loss probability of packets with both levels of drop precedence better than does PBS. We conducted simulation experiments focusing on the output port of a $16 \times 16$ photonic packet switch.

In the simulation, packets arrive at each input port of the photonic packet switch following a Bernoulli process with rate $\rho$. The ratio of the arrival rate 


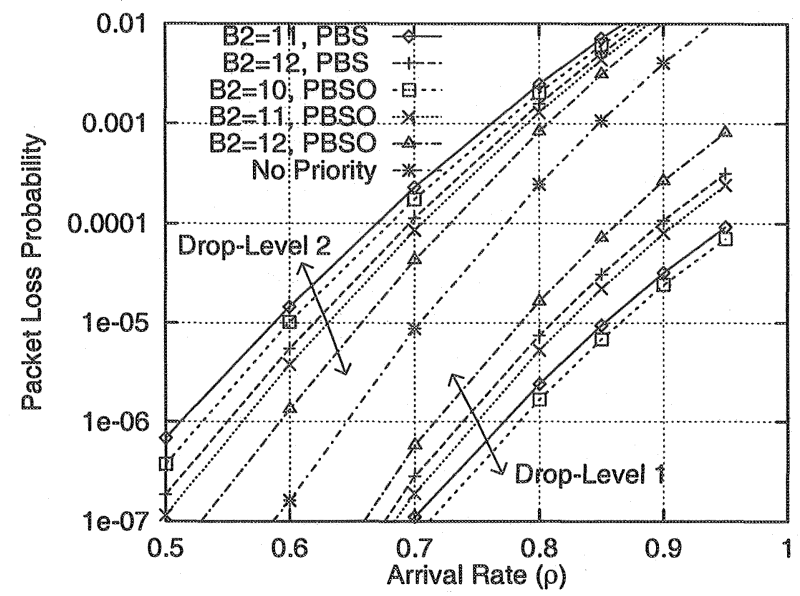

Figure 7. Packet loss probability of drop-level 1 and drop-level 2 packets (level 1: level 2=1:1, $B=15)$

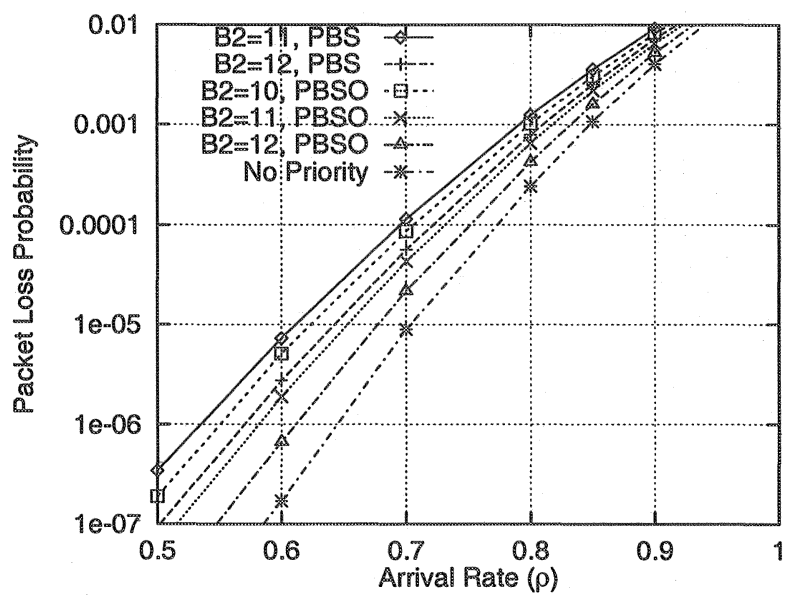

Figure 8. Mean packet loss probability (level 1:level 2=1:1, $B=15$ )

of drop-level 1 packets to that of drop-level 2 packets is set at $1: 1,3: 1$, and $1: 3$. The destinations of all arriving packets are assumed to be uniformly distributed through all the output ports. The number of ports is set at $N=16$. The simulation was run during $10^{9}$ packet generation time.

Figures 7 through 10 show the packet loss probabilities of PBS and PBSO. We also include the results for the case when all packets are forwarded without priority. The vertical axis shows the packet loss probability while the horizontal axis shows the total packet arrival rate of both drop-level 1 and drop-level 2 at each input port. Several threshold values are plotted: $B_{2}=11$ and 12 for PBS, $B_{2}=10,11$, and 12 for PBSO. 
Figure 7 shows packet loss probabilities of drop-level 1 and drop level 2 packets, where $50 \%$ of all the packets are drop-level 1 packets and the rest are drop-level 2 packets. Similarly to the results presented in [13], we find that PBS reduces the packet loss probability of drop-level 1 packets more than does non-priority packet forwarding. This reduction is achieved at the expense of the drop-level 2 packet loss probability. We also find that the performance of the PBSO strategy is similar to that of the PBS strategy. Although we omit the figure, the difference in performance for packets with different levels of drop precedence is also found in other maximum queue length, $B=20$ and 25. This indicates that both PBSO and PBS can provide different levels of drop precedence in DiffServ AF.

We now focus on the difference between PBS and PBSO, when the same threshold, $B_{2}$, is used in Fig. 7. The figure indicates that the performance of PBSO for the drop-level 2 packets is better than that of PBS. On the other hand, Fig. 7 shows that performance of PBSO for drop-level 1 packets is inferior to that of PBS. This is explained as follows. In contrast to the PBS strategy, PBSO allows drop-level 2 packets to enter the buffer even if the queue length is equal to or longer than the threshold. Some packets are not overwritten if drop-level 1 packets do not arrive before drop-level 2 packets go ahead the threshold. This indicates that the performance of PBSO for drop-level 2 packets is better than that of PBS. As for drop-level 1 packets in the PBSO strategy, when the queue length is equal to or longer than the threshold, drop-level 1 packets are not affected by drop-level 2 packets. However, once drop-level 2 packets that are not overwritten go ahead of the threshold, the packets cannot be overwritten. If many drop-level 1 packets arrive at the buffer at that time, the packets are likely to be discarded more easily than that in the PBS strategy. Although PBSO degrades the packet loss probability of drop-level 1 packets, PBSO improves the total packet loss probability as shown in Fig. 8. This indicates better buffer utilization in PBSO.

We next examine the difference between PBS and PBSO by using the different threshold values. From Fig. 7, we find the performance of PBSO for packets with both levels of drop precedence is slightly improved compared to that of PBS by proper determination of the threshold. For example, a pair of $B_{2}=12$ in PBS and $B_{2}=11$ in PBSO. Recall that since PBS and PBSO have a similar complexity, $\mathrm{PBSO}$ is also applicable to the management of optical buffers. PBSO also improves the total packet loss probability as shown in Fig. 8.

While PBS and PBSO provide different levels of drop precedence, their buffer utilization is lower than that of non-priority packet forwarding. Figure 8 compares the mean packet loss probabilities in PBS, PBSO, and non-priority cases. Here, the mean packet loss probability in PO is the same as that in the non-priority case. It means that the buffer utilization in PBS and PBSO is lower than that in the PO strategy. However, PBS and PBSO are effective because 


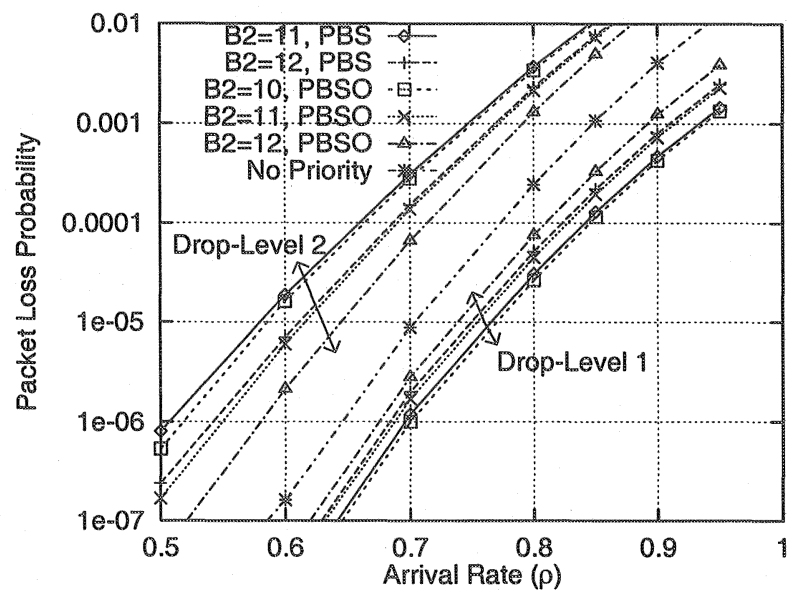

Figure 9. Packet loss probability of drop-level 1 and drop-level 2 packets (level 1:level 2=3:1, $B=15)$

an optical buffer requires high-speed management while PO's $O(B)$ is very complex.

Finally, we investigate difference in performance of PBSO and PBS depending on the ratio of the arrival rate of drop-level 1 packets to that of drop-level 2 packets. Figure 9 shows packet loss probabilities of drop-level 1 packets and drop-level 2 packets, in which $75 \%$ of the packets is drop-level 1 and the rest are drop-level 2. We find that in both PBS and PBSO strategies, the difference between the packet loss probabilities of drop-level 1 and drop-level 2 packets is smaller than in the cases shown in Fig. 7. Accordingly, the difference between PBS and PBSO also becomes smaller. Figure 10 shows the packet loss probabilities of drop-level 1 and drop-level 2 packets, where $25 \%$ of all the packets are drop-level 1 and the rest are drop-level 2. We find that the difference between the packet loss probabilities of drop-level 1 and drop-level 2 packets become larger than in the cases in Fig. 7. More notably, we find that the performance of PBSO for both levels of drop precedence is improved when compared to that of PBS, by proper determination of the threshold. For example, a pair of $B_{2}=12$ in PBS and $B_{2}=11$ in PBSO. The packet loss probability of drop-level 1 packets in PBSO is approximately 50\% lower than that in PBS. We therefore conclude that $\mathrm{PBSO}$ provides better performance than PBS with a smaller ratio of the arrival rate of drop-levell 1 packets to that of drop-level 2 packets.

\section{Concluding Remarks}

We investigated the management of an optical buffer providing different levels of drop precedence in DiffServ AF. Since a photonic packet switch requires high-speed buffer management as well as high-speed address-lookup function, 


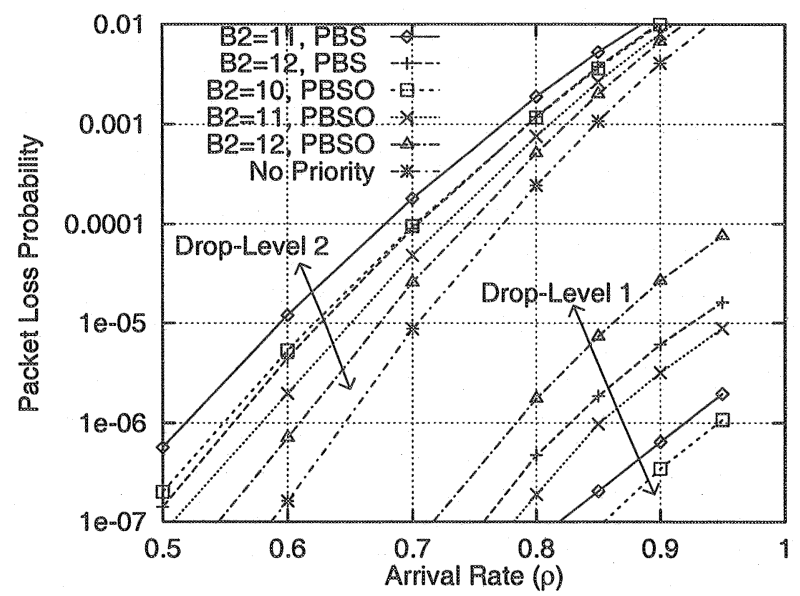

Figure 10. Packet loss probability of drop-level 1 and drop-level 2 packets (level 1:level 2=1:3, $B=15$ )

a less complex strategy should be adopted. We described the implementation of PBS, a strategy developed for providing DiffServ AF. We showed that PBS is applicable to single-stage optical buffers. We also proposed the PBSO strategy, which is applicable to broadcast-and-select-based single-stage optical buffers. PBSO is also applicable to existing space-switch-based optical buffers with an addition of a few optical components. The PBS and PBSO strategies are both based on a single queue and their complexities are $O(1)$ and $O(p)$, respectively, where $p$ is the number of levels of drop precedence. Through simulation study, we found that both strategies provide different levels of drop precedence. PBSO improves buffer utilization and packet loss probabilities in both levels of drop precedence. The amount of improvement in the packet loss probability clearly depends on the ratio of the arrival rate of drop-level 1 packets to that of drop-level 2 packets: the smaller the ratio, the smaller the packet loss probability.

\section{References}

[1] S. Blake, D. Black, M. Carlson, E. Davies, Z. Wang, and W. Weiss. An architecture for Differentiated Services. RFC 2475, December 1998.

[2] S. L. Danielsen, B. Mikkelsen, C. Joergensen, T. Durhuus, and K. E. Stubkjaer. WDM packet switch architectures and analysis of the influence of tunable wavelength converters on the performance. IEEE/OSA Journal of Lightwave Technology, 15(2):219-227, February 1997.

[3] A. Demers, S. Keshav, and S. Shenker. Analysis and simulatin of a fair queueing algorithm. ACM SIGCOMM '89, 19(4):1-12, 1989.

[4] Juan Diao and Pak L. Chu. Analysis of partially shared buffering for WDM optical packet switching. IEEE/OSA Journal of Lightwave Technology, 17(12):2461-2469, December 1999. 
[5] Sally Floyd and Van Jacobson. Link-sharing and resource management models for packet networks. IEEE/ACM Transactions on Networking, 3(4), August 1995.

[6] Keishi Habara, Hiroaki Sanjo, Hideki Nishizawa, Yoshiaki Yamada, Shigeki Hino, Ikuo Ogawa, and Yasumasa Suzaki. Large-capacity photonic packet switch prototype using wavelength routing techniques. IEICE Transactions on Communications, E83B(10):2304-2311, October 2000.

[7] J. Heinanen, F. Baker, W. Weiss, and J. Wroclawski. Assured Forwarding PHB groups. RFC 2597, June 1999.

[8] David K. Hunter and Ivan Andonovic. Approaches to optical Internet packet switching. IEEE Communications Magazine, 38(9):116-122, September 2000.

[9] David K. Hunter, Meow C. Chia, and Ivan Andonovic. Buffering in optical packet switches. IEEE/OSA Journal of Lightwave Technology, 16(12):2081-2094, December 1998.

[10] David K. Hunter, W. David Cornwell, Tim H. Gilfedder, André Franzen, and Ivan Andonovic. SLOB: A switch with large optical buffers for packet switching. IEEE/OSA Journal of Lightwave Technology, 16(10):1725-1736, October 1998.

[11] Kenichi Kitayama and Naoya Wada. Photonic IP routing. IEEE Photonic Technology Letters, 11(12):1689-1691, December 1999.

[12] L. Kleinrock. Queueing systems Volume II: Computer applications. A Wiley-Interscience Publication, 1976.

[13] Y. Lin and J. A. Silverster. Priority queueing strategies and buffer allocation protocols for traffic control at an ATM integrated broadband switching system. IEEE Journal on Selected Areas in Communications, 9(9):1524-1536, December 1991.

[14] M. Shreedhar and George Varghese. Efficient fair queueing using deficit round robin. ACM Computer Communication Review, 25(4):231-242, October 1995.

[15] Naoya Wada, Hiroaki Harai, Wataru Chujo, and Fumito Kubota. Photonic packet routing based on multi-wavelength label switching using fiber Bragg gratings. In Proceedings of ECOC 2000 (26th European Conference on Optical Communication), volume 4 (No. 10.4.6), pages 71-72, September 2000. 